\title{
Long-Time Survival of a Patient with Metastatic Pancreatic Cancer: A Case Report
}

\author{
Željko Soldić Daniela Žitnjak Ante Bolanča \\ Zvonko Kusić
}

Department of Oncology and Nuclear Medicine, Clinical Hospital Center Sisters of Mercy, Zagreb, Croatia

\section{Key Words}

Cancer · Pancreas · Liver metastases · Cisplatin

\begin{abstract}
Pancreatic cancer is a malignant neoplasm of the pancreas. It does not cause any symptoms in the early stage, and later symptoms are nonspecific, thus the disease is usually diagnosed when already advanced. In 2008, pancreatic cancer ranked eighth on the list of the 10 most common cancers among men in Croatia and tenth on the list of the most common cancers among Croatian women. Pancreatic cancer has a poor prognosis, with a survival time of only 6-8 months for metastatic disease. Gemcitabine is the standard chemotherapeutic option. Other chemotherapeutic agents include 5 -fluorouracil and leucovorin. In this paper, we present a case of a patient diagnosed with locally advanced and metastatic pancreatic cancer, who is still alive and currently receives his fourth line of chemotherapy 5 years after the diagnosis. Following disease progression on gemcitabine chemotherapy, he was treated with chemoradiotherapy which, however, had no effect. We then applied cisplatin monochemotherapy which offered excellent disease control, was well tolerated by the patient and, although somewhat obsolete in this form, showed to be a valuable chemotherapeutic option.
\end{abstract}

\section{Introduction}

Pancreatic cancer is a malignant neoplasm of the pancreas. Endocrine pancreatic tumors account for only about $1 \%$ of all cases. In contrast, exocrine tumors are far more common, with $95 \%$ of them being adenocarcinomas while the remaining $5 \%$ include adenosquamous carcinomas, signet ring cell carcinomas, hepatoid carcinomas, colloid carcinomas, undifferentiated carcinomas, and undifferentiated carcinomas with osteoclast-like giant cells [1]. Because pancreatic cancer does not cause any symptoms in 
the early stage and the later symptoms are nonspecific and vary, the disease is usually diagnosed when already advanced and, thus, the survival is poor. Each year, approximately 32,000 patients are diagnosed with pancreatic cancer in the United States, and approximately 31,000 patients die from it each year, making pancreatic cancer the fourth leading cause of cancer-related death [2]. In 2008, with an incidence rate of $374 / 100,000$ and a mortality rate of $363 / 100,000$, pancreatic cancer ranked eighth on the list of the 10 most common cancers among men in Croatia [3]. The incidence and mortality rates of pancreatic cancer among Croatian women were somewhat lower, with 349 and 316 per 100,000, respectively, making it number 10 on the list of the most common cancers [3].

According to the analysis of 684 patients treated for pancreatic cancer in the Memorial Sloan-Kettering Cancer Center, the median survival time for patients with pancreatic ductal carcinoma who underwent resection was 14.3 months, but only 4.9 months for unresectable patients [4]. Gemcitabine is the standard chemotherapy option [5]. Capecitabine has been shown to provide long-term survival (approx. 4 years) in 2 patients with metastatic pancreatic cancer after gemcitabine treatment failure, possibly due to its higher activity of thymidine phosphorylase, an enzyme that activates 5 -fluorouracil (5-FU) in tumor tissues, and thus its higher thymidine phosphorylase/dihydropyrimidine dehydrogenase ratio which could predict survival benefit with 5-FU-based agents [6].

\section{Case Report}

A 42-year-old male with jaundice, weight loss, nocturnal upper right quadrant abdominal pain, diarrhea and postprandial epigastric pain was hospitalized after ultrasonically suspected infiltration in the head of the pancreas. An abdominal CT scan showed an expansive lesion retropancreatically, between the pancreas and inferior vena cava and below the portal vein, measuring $41 \times 25 \mathrm{~mm}$, with enlarged para-aortic and interaorticocaval lymph nodes, a dilated intra- and extrahepatic biliary tree and stenosis of the common bile duct. An endoscopic retrograde cholangiopancreatography was performed, and the patient was referred to an abdominal surgeon. During explorative laparotomy, the tumor was found to be inoperable because of local vascular infiltration and liver metastases.

Pathohistological diagnosis confirmed the clinical diagnosis.

One month later, chemotherapy with gemcitabine was started. After 6 cycles of chemotherapy (i.e. 22 applications), a CT scan showed no liver metastases; however, the tumor in the head of the pancreas was still visible and unchanged in size. The patient was again referred to a surgeon who declined the possibility of an operative procedure. Therefore, chemoradiotherapy was started during which the patient received the planned dose of $41.4 \mathrm{~Gy}(23 \times 1.8 \mathrm{~Gy})$ simultaneously with monthly intravenous 5-FU (5-day chemotherapy, 6 cycles). CT monitoring of the tumor response to chemoradiotherapy showed a relapse of the liver metastases, with no change in the size of the primary tumor or enlarged abdominal lymph nodes. Thus, a third line of chemotherapy with cisplatin monotherapy was started. After 5 cycles, a CT scan revealed stationary liver lesions and an unchanged primary tumor. The disease remained stable for 18 months until the patient had to be hospitalized for high blood glucose levels. Along with the diagnosis of insulin-dependent diabetes mellitus, new liver metastases were found. Five years after the diagnosis, the patient is still alive and receives the fourth line of chemotherapy (5-FU and leucovorin).

\section{Discussion}

The prognosis for patients with metastatic pancreatic cancer is extremely poor, with a median survival time of 3-6 months [2]. The standard cytotoxic treatment is gemcitabine [5]. However, the addition of other agents such as cisplatin, irinotecan, oxaliplatin, 
taxanes and biologically targeted agents has shown higher response rates [7]. According to a recently published meta-analysis, gemcitabine in combination with capecitabine or oxaliplatin is associated with improved overall survival and overall response rates in locally advanced and metastatic pancreatic cancer patients, while the gemcitabine/ cisplatin combination showed no survival benefit compared to the gemcitabine monotherapy [8].

For refractory pancreatic cancer, however, there is no consensus regarding the optimal treatment. Oettle et al. [9], when they evaluated FOLFOX as a second-line treatment in advanced pancreatic cancer, found that chemotherapy offers better overall survival to refractory patients as compared to best supportive care. According to the final results of the CONKO-003 trial, the addition of oxaliplatin to 5-FU and leucovorin improves overall survival and progression-free survival when compared to treatment with 5-FU and leucovorin alone [10]. Some studies have demonstrated the efficacy of gemcitabine/ oxaliplatin combination chemotherapy as second-line treatment [11]. Combination of oxaliplatin and capecitabine after treatment failure with gemcitabine has shown some activity [6]. Paclitaxel as monotherapy has considerable efficacy and low toxicity in second-line treatment [12], as does FOLFIRI [13]. Bevacizumab and erlotinib combination showed poor results [14], as did the use of sunitinib as a single agent [15]. Overall, little progress has been made so far in the field of refractory pancreatic cancer treatment, and there is no evidence-based treatment recommended for these patients.

Cisplatin monotherapy is seldom considered in modern treatment of refractory pancreatic cancer, although it is a widely used chemotherapeutic drug and the first member of a class of platinum-containing anticancer drugs. The number of patients initially responding to this therapy is high; however, the majority of cancer patients eventually relapse with cisplatin-resistant disease. According to our possibilities and clinical experience, we introduced cisplatin administered by bi-weekly infusion as third-line chemotherapy in our patient. This resulted in a remarkable response, providing 18 months of disease stability and contributing to a 5-year survival in an initially unresectable patient with liver and lymph node metastases. Thus, although today surpassed by new exciting drugs and their combinations, cisplatin monotherapy should be considered in refractory pancreatic cancer patients with satisfactory serum creatinine levels. It represents a valuable option in these patients, especially when one is faced with non-medical limitations in form of means restriction. 


\section{References}

-1 Yao JC, Eisner MP, Leary C, Dagohoy C, Phan A, Rashid A, Hassan M, Evans DB: Population-based study of islet cell carcinoma. Ann Surg Oncol 2007;14:3492-3500.

2 Jemal A, Siegel R, Ward E, Hao Y, Xu J, Thun MJ: Cancer statistics, 2009. CA Cancer J Clin 2009;59:225-249.

3 Croatian National Institute of Public Health. www.hzjz.hr.

4 Conion KC, Klimstra DS, Brennan MF: Long-term survival after curative resection for pancreatic ductal adenocarcinoma. Ann Surg 2007;223:273-279.

-5 Carmichael J, Fink U, Russel RC, Spittle MF, Harris AL, Spiesi G, Blatter J: Phase II study of gemcitabine in patients with advanced pancreatic cancer. Br J Cancer 1996;73:101-105.

6 Saif MW, Kang SP, Ledbetter L, Steg A, Diasio R, Johnson M: Long-term survival on capecitabine in two gemcitabine refractory pancreatic cancer patients. Is there a pharmacogenetic explanation? JOP 2007;8:799805.

7 Stathopoulos GP, Androulakis N, Souglakos J, Stathopoulos J, Georgoulias V: Present treatment and future expectations in advanced pancreatic cancer. Anticancer Res 2008;28:1303-1308.

8 Hu J, Zhao G, Wang HX, Tang L, Xu YC, Ma Y, Zhang FC: A meta-analysis of gemcitabine containing chemotherapy for locally advanced and metastatic pancreatic adenocarcinoma. J Hematol Oncol 2011;4:11.

9 Oettle H, Pelzer U, Stieler J, Hilbig A, Roll L, Schwaner I, et al: Oxaliplatin/folinic acid/5-fluorouracil (24 h $(\mathrm{OFF})$ ) plus best supportive care versus best supportive care alone (BSC) in second-line therapy of gemcitabine-refractory advanced pancreatic cancer (CONKO 003). J Clin Oncol 2005;23(16 suppl):abstract 4031.

10 Pelzer U, Kubica K, Stieler J, Schwaner I, Heil G, Gorner M, et al: A randomized trial in patients with gemcitabine refractory pancreatic cancer. Final results of the CONKO 003 study. J Clin Oncol 2008;26(15 suppl):abstract 4508.

-11 Demols A, Peeters M, Polus M, Marechal R, Gay F, Monsaert E, Hendlisz A, Van Laethem JL: Gemcitabine and oxaliplatin (GEMOX) in gemcitabine refractory advanced pancreatic adenocarcinoma: a phase II study. $\mathrm{Br}$ J Cancer 2006;94:481-485.

12 Oettle H, Arnold D, Esser M, Huhn D, Riess H: Paclitaxel as weekly second-line therapy in patients with advanced pancreatic carcinoma. Anticancer Drugs 2000;11:635-638.

-13 Yoo C, Hwang JY, Kim JE, Kim TW, Lee JS, Park DH, Lee SS, Seo DW, Lee SK, Kim MH, Han DJ, Kim SC, Lee JL: A randomised phase II study of modified FOLFIRI.3 versus modified FOLFOX as second-line therapy in patients with gemcitabine-refractory advanced pancreatic cancer. Br J Cancer 2009;101:1658-1663.

-14 Ko AH, Venook AP, Bergsland EK, Kelley RK, Korn WM, Dito E, Schillinger B, Scott J, Hwang J, Tempero MA: A phase II study of bevacizumab plus erlotinib for gemcitabine-refractory metastatic pancreatic cancer. Cancer Chemother Pharmacol 2010;66:1051-1057.

-15 O'Reilly EM, Niedzwiecki D, Hall M, Hollis D, Bekaii-Saab T, Pluard T, Douglas K, Abou-Alfa GK, Kindler HL, Schilsky RL, Goldberg RM, Cancer and Leukemia Group B: A Cancer and Leukemia Group B phase II study of sunitinib malate in patients with previously treated metastatic pancreatic adenocarcinoma (CALGB 80603). Oncologist 2010;15:1310-1319. 